Advanced divertor configurations with large flux expansion.

V. A. Soukhanovskii et. al

June 12, 2012

Journal of Nuclear Materials 
This document was prepared as an account of work sponsored by an agency of the United States government. Neither the United States government nor Lawrence Livermore National Security, LLC, nor any of their employees makes any warranty, expressed or implied, or assumes any legal liability or responsibility for the accuracy, completeness, or usefulness of any information, apparatus, product, or process disclosed, or represents that its use would not infringe privately owned rights. Reference herein to any specific commercial product, process, or service by trade name, trademark, manufacturer, or otherwise does not necessarily constitute or imply its endorsement, recommendation, or favoring by the United States government or Lawrence Livermore National Security, LLC. The views and opinions of authors expressed herein do not necessarily state or reflect those of the United States government or Lawrence Livermore National Security, LLC, and shall not be used for advertising or product endorsement purposes. 


\section{Advanced divertor configurations with large flux expansion ${ }^{\text {负 }}$}

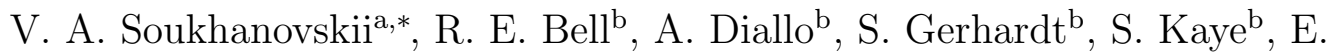
Kolemen $^{\mathrm{b}}$, B. P. LeBlanc ${ }^{\mathrm{b}}$, A. McLean ${ }^{\mathrm{a}}$, J. E. Menard ${ }^{\mathrm{b}}$, S. F. Paul ${ }^{\mathrm{b}}$, M.

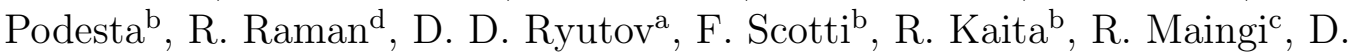
M. Mueller ${ }^{\mathrm{b}}$, A. L. Roquemore ${ }^{\mathrm{b}}$, H. Reimerdes ${ }^{\mathrm{e}}$, G.P. Canal ${ }^{\mathrm{e}}$, B. Labit ${ }^{\mathrm{e}}$, W.

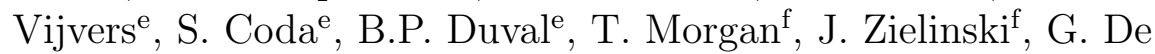

Temmerman ${ }^{\mathrm{f}}$, B. Tal ${ }^{\mathrm{g}}$

${ }^{a}$ Lawrence Livermore National Laboratory, Livermore, CA, USA

${ }^{b}$ Princeton Plasma Physics Laboratory, Princeton, NJ, USA

${ }^{c}$ Oak Ridge National Laboratory, Oak Ridge, TN, USA

${ }^{d}$ University of Washington, Seattle, WA, USA

${ }^{e}$ Ecole Polytechnique Fédérale de Lausanne, Centre de Recherches en Physique des

Plasmas, Association Euratom Confédération Suisse, Lausanne, Switzerland

${ }^{f}$ FOM Institute for Plasma Physics Rijnhuizen, Association EURATOM-FOM, Trilateral

Euregio Cluster, Postbus 1207, 3430BE, Nieuwegein, The Netherlands

${ }^{g}$ WIGNER Research Centre for Physics, Budapest, Hungary

\section{Abstract}

Experimental studies of the novel snowflake divertor concept (D. Ryutov, Phys. Plasmas 14, 064502 (2007)) performed in the NSTX and TCV tokamaks are reviewed in this paper. The snowflake divertor enables power sharing between divertor strike points, as well as the divertor plasma-wetted area, effective connection length and divertor volumetric power loss to increase beyond those in the standard divertor, potentially reducing heat flux and plasma temperature at the target. It also enables higher magnetic shear

\footnotetext{
Paper I-12

*Corresponding author. Address: P. O. Box 808, L-637, Lawrence Livermore National Laboratory, Livermore, CA 94551, USA. Email: vlad@llnl.gov
} 
inside the separatrix, potentially affecting pedestal MHD stability. Experimental results from NSTX and TCV confirm the predicted properties of the snowflake divertor. In the NSTX, a large spherical tokamak with a compact divertor and lithium-coated graphite plasma-facing components (PFCs), the snowflake divertor operation led to reduced core and pedestal impurity concentration, as well as re-appearance of Type I ELMs that were suppressed in standard divertor $\mathrm{H}$-mode discharges. In the divertor, an otherwise inaccessible partial detachment of the outer strike point with an up to $50 \%$ increase in divertor radiation and a peak divertor heat flux reduction from 3-7 $\mathrm{MW} / \mathrm{m}^{2}$ to $0.5-1 \mathrm{MW} / \mathrm{m}^{2}$ was achieved. Impulsive heat fluxes due to TypeI ELMs were significantly dissipated in the high magnetic flux expansion region. In the TCV, a medium-size tokamak with graphite PFCs, several advantageous snowflake divertor features (cf. the standard divertor) have been demonstrated: an unchanged L-H power threshold, enhanced stability of the peeling-ballooning modes in the pedestal region (and generally an extended second stability region), as well as an H-mode pedestal regime with reduced (× 2-3) Type I ELM frequency and slightly increased (20-30\%) normalized ELM energy, resulting in a favorable average energy loss comparison to the standard divertor. In the divertor, ELM power partitioning between snowflake divertor strike points was demonstrated. The NSTX and TCV experiments are providing support for the snowflake divertor as a viable solution for the outstanding tokamak plasma-material interface issues.

Keywords: JNM keywords: P0500 Plasma-Materials Interaction, P0600

Plasma Properties

PSI-20 keywords: Divertor, Divertor plasma, Power deposition, ELM, TCV, 


\section{NSTX}

PACS: 52.55.Fa Tokamaks, spherical tokamaks, 52.55.Rk Power exhaust; divertors

\section{Introduction}

The present vision of the tokamak plasma-material interface is an axisymmetric magnetic X-point divertor. The standard X-point divertor configuration enables intense heat and particle fluxes from the core plasma to be directed to a separate divertor chamber for special handling. The envisaged handling strategies include partitioning the SOL power $P_{S O L}$ between inner, outer, lower and upper divertor legs, reducing parallel heat and particle fluxes through divertor volumetric loss processes, and reducing heat flux $q_{\perp}$ deposited on the plasma facing components (PFCs) via the increased plasmawetted area $A_{w}[1,2]$. Divertor geometry, in particular, has been known as a key factor for divertor performance optimization $[1,3,4]$. The ITER divertor design, a closed divertor with tilted vertical targets and a partial radiative detachment of the strike points, represents an optimized standard divertor geometry based on experimental tokamak studies, theory and modeling developments over the last two decades $[1,2,5]$. However, for the proposed advanced tokamak and spherical tokamak (ST) based fusion nuclear science facilities $[6,7]$ and for the DEMO [8], the standard divertor solution is insufficient since the expected heat fluxes would exceed the presently allowed steady-state limit of 5-10 MW/m² and ELM-like transients 0.1-0.5 MJ/m².

In addition to the divertor hardware geometry optimization that includes divertor target plate positioning, e.g., horizontal or vertical orienta- 
tion, poloidal (at angle $\alpha$ ) or toroidal tilting of target elements, and a closed divertor, it is also possible to optimize the divertor magnetic configuration. The impact of divertor magnetic flux geometry on target particle and heat flux profiles has been verified in tokamak experiments $[3,4,9]$. The main features that can be optimized are the X-point to strike point distance (parallel $L_{X}$ or poloidal $L_{p o l}$ length affecting divertor volume, neutral penetration and increasing radial heat diffusion) and poloidal magnetic flux expansion $f_{\text {exp }}$. The flux expansion is defined as $f_{\text {exp }}=\left(B_{p} / B_{t o t}\right)_{M P}\left(B_{p} / B_{t o t}\right)_{S P}^{-1}$, where $B_{p}$ is the poloidal magnetic field, and $B_{t o t}$ is the total magnetic field, both evaluated at the mid-plane separatrix $\left(R_{M P}\right)$ and at the strike point $\left(R_{S P}\right)$. Increased $f_{\text {exp }}$ leads to an increased flux tube volume and to the increased plasma-wetted area $A_{w}=2 \pi R_{S P} f_{\text {exp }} \lambda_{q} / \sin \alpha$, where $\lambda_{q}$ is the mid-plane SOL power width. While $\lambda_{q}$ is a fundamental SOL parameter determined by plasma transport and pedestal MHD stability [10], the geometry parameters $R_{S P}, f_{\text {exp }}, \alpha$, etc are constrained by the geometry and design of the vacuum vessel and poloidal field coils.

Recently, new divertor magnetic geometry concepts have emerged: the Super-X (SX) divertor [11] and the snowflake (SF) divertor [12] configurations. Both concepts enable the divertor plasma-wetted area, effective connection length and divertor volumetric power loss to increase beyond those in the standard divertor, potentially reducing heat flux and plasma temperature at the target. The SX divertor concept is being implemented in the MAST Upgrade tokamak [13], and initial physics experiments are being conducted at DIII-D [14]. This paper summarizes experimental SF divertor configuration studies performed in the National Spherical Torus Experiment (NSTX) and 
the Tokamak à Configuration Variable (TCV). The results demonstrate that the SF divertor may not only hold promise for the solution of the outstanding plasma-material interface issues, but could also be used as a laboratory for pedestal stability and divertor heat transport studies in existing tokamaks.

The SF magnetic configuration uses a second-order null-point created by bringing two first-order null-points of the standard divertor together $[12,15$, 16]. Poloidal magnetic flux surfaces in the vicinity of the second-order null point have hexagonal separatrix branches with an appearance of a snowflake. In the tokamak, two or more existing divertor coils can be used to obtain an ideal SF configuration, as well as its two derivative configurations: a SF-plus and a SF-minus. In the SF-plus configuration the divertor coil currents slightly exceed those of the ideal SF case resulting in the disconnected secondary null-point located in the private flux region. In the SF-minus con-

figuration, the corresponding divertor coil currents are slightly lower, and the second null-point located on the main separatrix, or, as in the asymmetric SF-minus, in the common flux region [16]. The deviation of these configuration from the ideal SF is described by the parameter $\sigma=d / a$, where $d$ is the distance between the null-points and $a$ is the plasma minor radius.

\section{Experiment}

The SF divertor concept has been studied in the NSTX and TCV tokamaks. NSTX is a large spherical tokamak with a major radius $R=0.85$ $\mathrm{m}$ and minor radius $a=0.67 \mathrm{~m}$ [17]. The NSTX divertor plate geometry is up-down symmetric and open, enabling flexibility in plasma and divertor shaping. Graphite tiles with evaporated lithium coatings (up to $200 \mathrm{mg}$ per 
discharge) were used as divertor PFCs. The SF divertor studies were performed in $I_{p}=0.8-1.0 \mathrm{MA} 4 \mathrm{MW}$ neutral beam injection (NBI) heated $\mathrm{H}$-mode discharges. The ion $\nabla B$ drift direction was toward the lower $\mathrm{X}$ point, and the toroidal field was $B_{t}=0.45 \mathrm{~T}$. Three existing lower divertor coils with currents in the $0.5-5 \mathrm{kA}$ range were used to obtain steady-state SF configurations $[18,19,20]$.

TCV is a medium-size conventional aspect ratio tokamak with $R=0.88$ $\mathrm{m}, a=0.25 \mathrm{~m}$, and $B_{t} \leq 1.5 \mathrm{~T}$ [21]. It has an open, up-down symmetric divertor plate geometry and graphite PFCs. Up to 16 poloidal field coils are available for plasma shape control, making it an ideal test bed for SF studies [22]. The SF experiments were conducted in 0.3 MA L- and H-mode discharges with up to $2 \mathrm{MW}$ of electron cyclotron heating (ECH) [22, 23, 24]. The ion $\nabla B$ drift direction was generally toward the lower divertor.

\section{Results and discussion}

The SF geometry properties and their benefits for pedestal stability and divertor heat flux mitigation have been confirmed in the complementary NSTX and TCV experiments. In both tokamaks, thanks to the open divertor geometry and a flexible plasma control system, ideal SF, SF-plus and SF-minus configurations were obtained [19, 20, 22, 21]. In TCV, SF-plus

configurations with $\sigma \simeq 0.5$ were used to study H-mode access, pedestal stability, ELMs and divertor properties. In NSTX, asymmetric SF-minus configurations with $\sigma \simeq 0.4-0.5$ were used to study pedestal characteristics and divertor heat flux mitigation between and during ELMs. 
Magnetic properties and control. The SF magnetic properties predicted theoretically [12] have been realized in NSTX and TCV experiments. Shown in Fig. 1 are the poloidal coil layouts, and the SF configurations obtained in NSTX and TCV. In both tokamaks, these SF configurations were compatible with a range of plasma triangularities and elongations that could be achieved with the standard divertor. A large region of very low $B_{p}$ in the SF nulls vicinity can be seen in the poloidal magnetic field distribution plot in Fig. 1. The region is larger than in the standard divertor X-point configuration, and it extends not only throughout the divertor separatrix branches (legs), but also deeper inside the separatrix (w.r.t. standard divertor). In $\mathrm{TCV}$, this resulted in increased null-region poloidal magnetic flux expansion by a factor 2-5, and a similar increase in the connection length, both within a radial extent of $1 \mathrm{~mm}$ of the mid-plane SOL [24]. The highest increase in $f_{\text {exp }}$ and $L_{\|}$in TCV was obtained with the ideal SF configuration. In NSTX, the asymmetric SF-minus configuration showed an up to 50-75 \% increase in $L_{\|}$and $A_{w}\left(f_{\text {exp }}\right)$ in the strike point region. The high $f_{\exp }$ region extended throughout $30-50 \%$ of the SOL width.

In both the NSTX and TCV tokamak experiments, steady-state SF configurations were obtained with pre-programmed divertor coil currents, i.e. without plasma control system feedback control.

Core and pedestal properties. The presence of a second poloidal field null in the vicinity of the separatrix may lead to increased magnetic shear inside the separatrix that can in turn affect the edge turbulence and H-mode confinement. For example, in conventional and spherical tokamaks, the L-H power threshold is significantly lower in a double null configuration (where the sec- 
ond upper X-point is on the same flux surface as the lower one), and the L-H power threshold depends on the X-point distance to the divertor target. The higher magnetic shear just inside the separatrix predicted for the SF configuration can also lead to stronger stabilization of ideal current-driven peeling modes and pressure-driven balooning MHD modes [15, 25]. Therefore, it was not clear apriori how the SF configuration would affect the confined tokamak plasma.

In NSTX, the SF configuration was compatible with high confinement plasma operation, with no degradation in H-mode core performance $[19,20]$. Core plasma parameters $\left(\bar{n}_{e}\right.$, central $\left.T_{e} \leq 1 \mathrm{keV}, \beta_{N} \leq 4.5\right)$ were similar to those in the standard divertor H-mode discharges. Similar high performance metrics of these discharges, e.g., $\tau_{E} \simeq 50-60 \mathrm{~ms}, W_{M H D} \simeq 200-250 \mathrm{~kJ}$, and the factor $H 98(y, 2) \simeq 1$ calculated using the TRANSP code, confirmed minimal, if any, impact of the snowflake phase on confinement. In these high-triangularity plasmas, the L-H transition power threshold was fairly low (about $1 \mathrm{MW}$ ), therefore no H-mode access studies were performed. The SF divertor phase had a profound effect on plasma impurity content: the total carbon inventory $N_{c}$ was reduced by 50-70\%. The observed reduction was attributed to the reduction of carbon physical sputtering fluxes in the SF divertor (due to very low divertor $T_{e}$ ), and to the particle expulsion effect from ELMs that appeared in the SF phase [20]. In the standard divertor Hmode discharge, lithium coatings on lower divertor PFCs reduced recycling and led to modified edge plasma pressure and current profiles and low- $n$ peeling-ballooning mode stabilization [26, 27], as the pedestal stability operating point was close to the peeling boundary. Depending on the lithium 
conditioning and other operational factors, ELMs could be completely or partially suppressed. The transition to the SF configuration led to a clear and reproducible destabilization of the ELMs. These large ELMs were classified as Type I, with somewhat irregular frequency of $f=12-35 \mathrm{~Hz}$ and $\Delta W_{M H D} / W_{M H D}$ in the range 5-10\%. The study of the ELM destabilization in the SF discharges requires clarification of the carbon role in pedestal pressure profiles and MHD stability calculations which are planned.

In TCV, H-mode threshold was systematically studied with a variation of $\mathrm{ECH}$ power in the range $0.25-1.5 \mathrm{MW}$ in otherwise similar discharges having the standard divertor and SF-plus configurations [23, 24]. The L-H transition power threshold was found to be similar in both cases over the (volumeaveraged) density range $3-7 \times 10^{19} \mathrm{~m}^{-3}$. Modest confinement improvement, up to $15 \%$, was noted in the SF-plus phase, albeit may be due to increased core shaping.

The SF-plus configuration in TCV had a profound effect on the pedestal stability [23, 24]. Shown in Fig. 2 are the time traces of a 1.5 MW EC-heated $\mathrm{H}$-mode discharge was started in the standard divertor configuration and in a later phase transitioned to the SF-plus configuration. The frequency of Type I ELMs decreased by 50-80 \% at the transition, while the energy loss per ELM event increased only by 20-30 \%. The SF-plus phase of the H-mode discharge therefore indicated improved performance with reduced energy lost through the ELM channel. Pedestal MHD stability calculations indicated that the stability operating point in both the standard divertor and the SF-plus was close to the kink-balooning stability boundary, and the SF-plus configuration was consistent with improved kink-ballooning stability [23, 24, 28]. Equilibria 
reconstructions also confirmed a higher magnetic shear inside the separatrix in the SF-plus phase.

SOL and divertor properties. The SF divertor geometry properties realized in NSTX and TCV had a significant impact on divertor heat and particle transport.

In NSTX, a significant between-ELM reduction of divertor peak heat flux was measured $[19,20]$. The SF-minus formation was always accompanied by a stable partial detachment of the outer strike point otherwise inaccessible in the standard divertor at $P_{S O L}=3 \mathrm{MW}[29,30]$. Fig. 3 illustrates the divertor time traces and divertor heat flux profiles in the standard divertor and SF divertor discharges. As the secondary null-point was formed and moved toward the primary X-point, the $\sigma$ parameter was continuously reduced. The divertor volume, $A_{w}$, and $L_{X}$ were continuously increased, resulting in measurable changes in divertor heat flux and radiated power. During the SF formation period that lasted for 100-200 ms, divertor power decreased from 1.8-2.0 MW to about 1.2 MW, and the peak heat flux was reduced from 4-7 MW/ $\mathrm{m}^{2}$ to $2-3 \mathrm{MW} / \mathrm{m}^{2}$ between ELMs. This decrease was interpreted as driven by both geometric changes in $L_{x}, A_{w}$ as well as some radiative losses. However, the reduction factor quickly exceeded the geometric factor proportional to $A_{w}$, as the estimated $q_{\|}$reduction from $100-115 \mathrm{MW} / \mathrm{m}^{2}$ in the standard divertor phase to $30-50 \mathrm{MW} / \mathrm{m}^{2}$ in the $\mathrm{SF}$ was observed. As the geometry continued to change, the SOL collisionality and volumetric losses increased further leading to a partial strike point detachment. The additional volumetric losses were corroborated by the divertor carbon radiation and the recombination rates that also continuously increased, exceeding that of the 
standard divertor by up to $50 \%$. After the partial detachment onset, the peak heat flux decreased further down to $0.5-1 \mathrm{MW} / \mathrm{m}^{2}$, while the total power received by the outer divertor decreased to below $1 \mathrm{MW}$. In spite of the formation of the highly-radiating detached region in the SF divertor, high core confinement was maintained for up to $10 \times \tau_{E}$ (i.e., up to a full duration of the SF phase 500-600 ms).

A significant reduction of steady-state divertor heat flux in the SF configuration is an encouraging news for future spherical tokamak based devices with inherently compact divertors. In previous NSTX divertor experiments, $q_{p k}$ showed a linear scaling with $P_{S O L}$ and a weak dependence on $n_{e}[29,30,31]$. Partial detachment of the outer strike point was obtained in H-mode discharges with the standard (although high $f_{\text {exp }}$ ) divertor using additional extrinsic $\mathrm{D}_{2}$ or $\mathrm{CD}_{4}$ puffing $[29,30]$. In the range of $\mathrm{SOL}$ power $1.5 \leq P_{S O L} \leq 5 \mathrm{MW}$, the outer strike point detachment did not occur without gas seeding because of insufficient divertor carbon $P_{\text {rad }}$ in the opengeometry, compact NSTX divertor. The peak heat flux reduction in the SF configuration (however, without any gas seeding) was similar to the $\mathrm{D}_{2}$-seeded partially detached divertor at $P_{S O L} \sim 3 \mathrm{MW}$. These results are summarized in Fig. 4. The operational window of the gas-seeded radiative divertor at high $P_{S O L}$ could be narrow as the required gas seeding rate could be incompatible with high pressure pedestal, ELM regime and X-point MARFE-free operation (e.g., [32]). Additional experimental work at higher power is needed to understand the limits of SF heat flux reduction due to geometric factors and volumetric losses. In NSTX, an additional $\mathrm{CD}_{4}$ or $\mathrm{D}_{2}$ seeding into the $\mathrm{SF}$ phase showed excellent divertor gas screening, increased divertor radiation, 
and stable MARFE-free operation (unaffected confinement). This suggests a way to enhance non-coronal impurity radiation in the SF configuration due to its already reduced $T_{e}$ regime.

Power partitioning due to heat diffusion between the separatrix branches and additional strike points in the SF configuration can be beneficial for steady-state divertor heat load handling, and perhaps even more critical for mitigating high transient heat and particle fluxes from Type I ELMs. Type I ELMs remain an unresolved issue for future divertor designs: ELM elimination techniques are explored, as radiative buffering of ELMs has been found ineffective [2]. Recent SF divertor theory and modeling developments highlighted two SF configuration effects on the transient ELM energy transport $[33,34]$. Reduced surface heating is expected due to the increased ELM energy deposition time $\tau_{E L M}$ and increased $A_{w}$ as the ELM convective ion heat pulse with energy $E_{E L M}$ travels over an increased field line length connecting the outer mid-plane and divertor target. The surface temperature rise is given by $\Delta T \sim E_{E L M} /\left(A_{w} \times \sqrt{\tau_{E L M}}\right)$. The second effect is the convective mixing of the ELM heat pulse in the null-point region leading to the heat flux partitioning between separatrix branches. These affects are being studied in the SF configurations in NSTX and TCV. In TCV, recent measurements using high spatial density Langmuir probe arrays [35] have confirmed the initial infrared thermography measurements $[23,24]$ of the heat and particle flux spreading into the additional strike points during Type I ELMs. The power partitioning between the SF separatrix branches was also observed in L-mode discharges, albeit at small values of $\sigma$ [36]. In the NSTX asymmetric SF-minus configuration, the heat fluxes from Type I ELMs were significantly 
dissipated, as shown in Fig. 5, from about $20 \mathrm{MW} / \mathrm{m}^{2}$ from an ELM in the standard divertor phase of the discharge, to $6-8 \mathrm{MW} / \mathrm{m}^{2}$ during the SF formation phase and eventually below $2 \mathrm{MW} / \mathrm{m}^{2}$ in the radiative $\mathrm{SF}$ phase. Peak target temperatures, measured by fast infrared thermography at peak ELM times, reached $1000-1200{ }^{\circ} \mathrm{C}$ in the standard divertor and only 300-500 ${ }^{\circ} \mathrm{C}$ in the SF phase (Fig. 3). Also apparent during ELMs was the emergence of an additional peak in the heat flux (and temperature) profile at the secondary separatrix location (and also where $f_{\text {exp }}$ and $L_{X}$ were similar to that of the standard divertor). Assuming that radiation was not a significant ELM power loss mechanism, the ELM peak heat flux reduction is consistent with the SF geometry and power sharing effects.

Modeling. Magnetic and plasma transport modeling generally show that the SF configuration can be achieved in some existing and future tokamaks with existing poloidal field coils and with reasonable currents, and the SF benefits for the pedestal stability and divertor heat load mitigation can indeed be realized. The magnetic configuration modeling is performed with GradShafranov plasma equilibrium codes and an electromagnetic model of the tokamak plasma and conductors, including the vessel and poloidal field coils. The modeling demonstrated feasibility of the SF configurations for NSTX [37, 19], TCV [22, 28], DIII-D [37], FDF [38], NSTX-U [39], and FAST [40]. Pedestal stability calculations performed with an ideal MHD stability code for TCV [28] showed an enhanced edge stability of the SF configuration, and similar conclusions were reached from the edge stability modeling for a DIIID-like SF equilibrium [25]. Two-dimensional multi-fluid transport models for simulated SF configurations [37, 38, 25] demonstrated that 1) the heat flux 
in the SF divertor was reduced with respect to the standard divertor and the reduction was stronger than just the ratio of the plasma-wetted areas; it also included the reduction due to increased volumetric losses; 2) the detachment threshold expressed in terms of the edge $n_{e}$ was lower in the SF divertor.

\section{Conclusions and outlook}

Experimental results from NSTX and TCV tokamaks suggest that the SF divertor configuration may be a viable divertor solution for present and future tokamaks. The present experiments demonstrated significant benefits of the SF divertor configuration, namely, steady-state and ELM divertor heat flux reduction via increased plasma-wetted area, volumetric losses, and power sharing between several strike points, as well as full compatibility with high-performance H-mode confinement and favorable edge stability modifications, in agreement with theory predictions. The SF divertor configuration apparently has a reduced divertor temperature, which can have a positive impact on target material erosion as well as on seeded impurity radiation enhancement. Much experimental research is still needed to fully qualify the SF divertor configuration as a reliable candidate for future high-power plasma-material interface. The areas for future SF studies include real-time

feedback control of the SF magnetic configuration, impurity production and transport, edge pedestal MHD stability and ELM regimes, effects of 3D magnetic perturbations on the edge stability and divertor fluxes, the role of divertor radiation, and compatibility of the SF with divertor cryogenic pumping. The SF configuration is being developed as a leading divertor heat flux mitigation candidate for NSTX Upgrade [39]. In NSTX-U, two up-down 
symmetric sets of four divertor coils will be used to test snowflake divertors for handling the projected steady-state peak divertor heat fluxes of 20-30 $\mathrm{MW} / \mathrm{m}^{2}$ in $2 \mathrm{MA}$ discharges up to $5 \mathrm{~s}$ long with up to $12 \mathrm{MW}$ NBI heating. Magnetic equilibria with SF configurations have been successfully modeled and showed that a robust snowflake control can be maintained even when time-dependent electromagnetic effects are included [39].

Acknowledgements. We thank the entire NSTX and TCV Teams for technical, engineering and computer support as well as plasma and diagnostic operations. This work was performed in part under the auspices of the U.S. Department of Energy under Contracts DE-AC52-07NA27344, DE-AC0209CH11466, DE-AC05-00OR22725, W-7405-ENG-36, DE-FG02-04ER54758 and the Swiss National Science Foundation.

[1] ITER Physics Expert Group on Divertor et al., Nucl. Fusion 39 (1999) 2391.

[2] LOARTE, A. et al., Nucl. Fusion 47 (2007) S203.

[3] LOARTE, A., J. Nucl. Mater. 290-293 (2001) 805.

[4] LOARTE, A., Plasma Phys. Control. Fusion 43 (2001) 183.

[5] LIPSCHUlTZ, B. et al., Nucl. Fusion 47 (2007) 1189.

[6] PENG, Y.-K. et al., Plasma Phys. Control. Fusion 47 (2005) 263.

[7] CHAN, V. et al., Nuclear Fusion 51 (2011) 083019.

[8] UNTERBERG, B. et al., This conference (2012). 
[9] LOARTE, A. et al., Nucl. Fusion 32 (1992) 681.

[10] EICH, T. et al., This conference (2012).

[11] VALAnJU, P. et al., Phys. Plasmas 16 (2009) 056110.

[12] RYUTOV, D., Phys. Plasmas 14 (2007) 064502.

[13] KATRAmados, I. et al., Fusion Eng. Des. 86 (2011) 1595.

[14] PETRIE, T. et al., This conference (2012).

[15] RYUTOV, D. et al., Phys. Plasmas 15 (2008) 092501.

[16] RYUTOV, D. et al., Plasma Phys. Control. Fusion 52 (2010) 105001.

[17] RAMAN, R. et al., Nucl. Fusion 51 (2011) 094011.

[18] SOUKHANOVSKII, V. et al., J. Nucl. Mater. 415 (2011) S365.

[19] SOUKHANOVSKII, V. A. et al., Nucl. Fusion 51 (2011) 012001.

[20] SOUKHANOVSKII, V. A. et al., Phys. Plasmas 19 (2012) 082504.

[21] CODA, S., Nucl. Fusion 51 (2011) 094017.

[22] PIRAS, F. et al., Plasma Phys. Control. Fusion 51 (2009) 055009.

[23] PIRAS, F. et al., Phys. Rev. Lett. 105 (2010) 155003.

[24] PIRAS, F. et al., Plasma Phys. Control. Fusion 52 (2010) 124010.

[25] UMANSKY, M. et al., Contrib. Plasma Phys. 50 (2010) 350.

[26] MANSFIELD, D. et al., J. Nucl. Mater. 390-391 (2009) 764. 
[27] MAINGI, R. et al., Phys. Rev. Lett. 103 (2009) 075001.

[28] MEDVEDEV, S. et al., Contrib. Plasma Phys. 50 (2010) 324.

[29] SOUKHANOVSKII, V. et al., Phys. Plasmas 16 (2009) 022501.

[30] SOUKHANOVSKII, V. et al., Nucl. Fusion 49 (2009) 095025.

[31] GRAY, T. et al., J. Nucl. Mater. 415 (2011) S360.

[32] GIROUD, C. et al., Nuclear Fusion 52 (2012) 063022.

[33] RYUTOV, D. D., Contrib. Plasma Phys. 52 (2012) 539.

[34] ROGNLIEN, T. et al., This conference (2012).

[35] LABIT, B. et al., Scrape-off layer properties of single-null and snowflake diverted plasmas in TCV, in Proceedings of the 38th EPS Conference on Plasma Physics, page P2.076, Strasbourg, France, 2011.

[36] REIMERDES, H. et al., Transport studies in the snowflake divertor in TCV, in 53rd Annual Meeting of the APS Division of Plasma Physics, November 14-18, 2011, Poster JP9.110.

[37] RYUTOV, D. et al., in Fusion Energy 2008 (Proc. 22nd Int. Conf. Geneva, 2008), CD-ROM file IC/P4-8, Vienna:IAEA.

[38] UMANSKY, M. et al., Nucl. Fusion 49 (2009) 075005.

[39] MENARD, J. et al., Nucl. Fusion 52 (2012) 083015. 
[40] CALABRO, G. et al., Snowflake divertor plasma studies on FAST proposal, in Proceedings of the 38th EPS Conference on Plasma Physics, page P1.066, Strasbourg, France, 2011. 
(a)

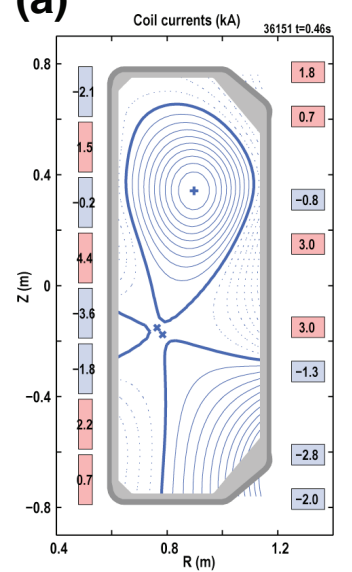

(c)

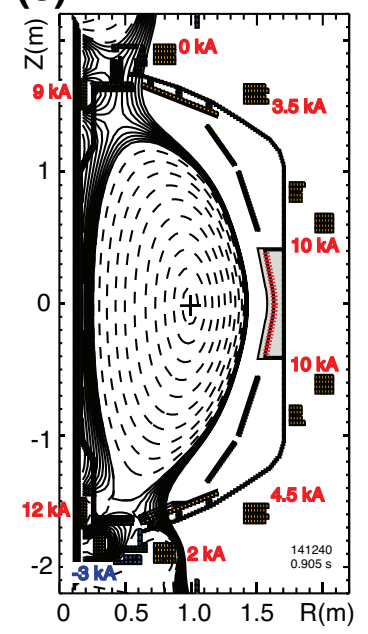

(b)

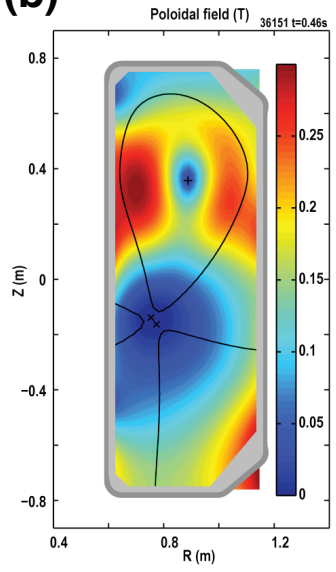

(d)
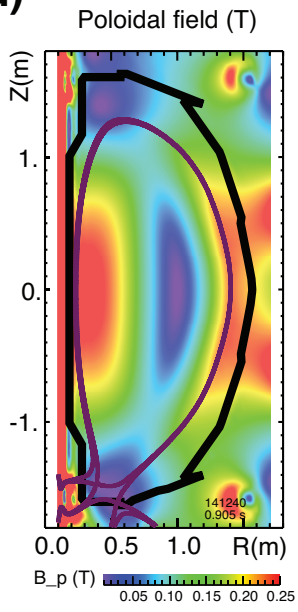

Figure 1: Poloidal cross-sections of the TCV (a),(b) $\left(I_{p}=0.3 \mathrm{MA}\right)$ and NSTX (c),(d), $\left(I_{p}=0.9 \mathrm{MA}\right)$ tokamaks indicating magnetic coil geometry and poloidal magnetic flux surfaces (a),(c) as well as the poloidal magnetic field strength $B_{p}(\mathrm{~b}),(\mathrm{d})$ in the snowflake divertor configurations. Note poloidal field coil currents expressed in kA. 


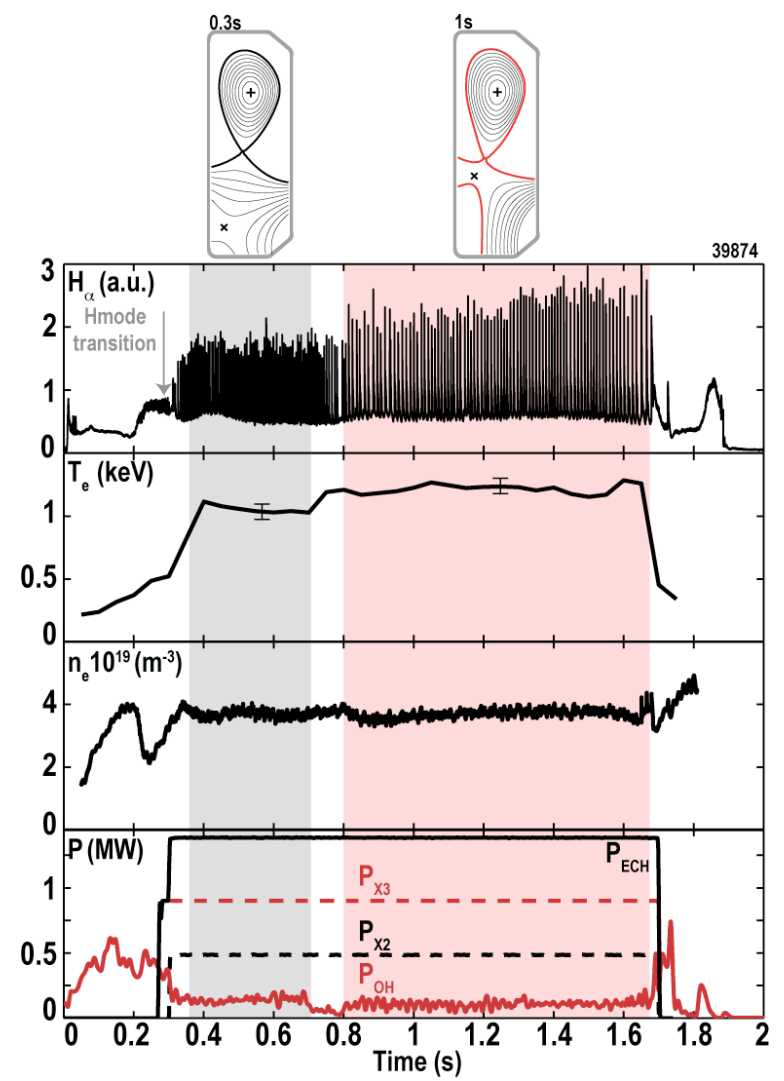

Figure 2: H-mode discharge time traces in TCV. (a) Edge $\mathrm{H}_{\alpha}$ intensity; (b) volumeaveraged $T_{e}$; (c) line-averaged $n_{e} ;(\mathrm{d})$ input power: ohmic-solid red line, second ECH harmonic (X2) - dashed black line, third ECH harmonic (X3) - dashed red line. The transition from the standard divertor to SF-plus occurs at about $0.8 \mathrm{~s}$. Reproduced with permission from F. Piras et al, Phys. Rev. Lett. 105 (2010) 155003. 

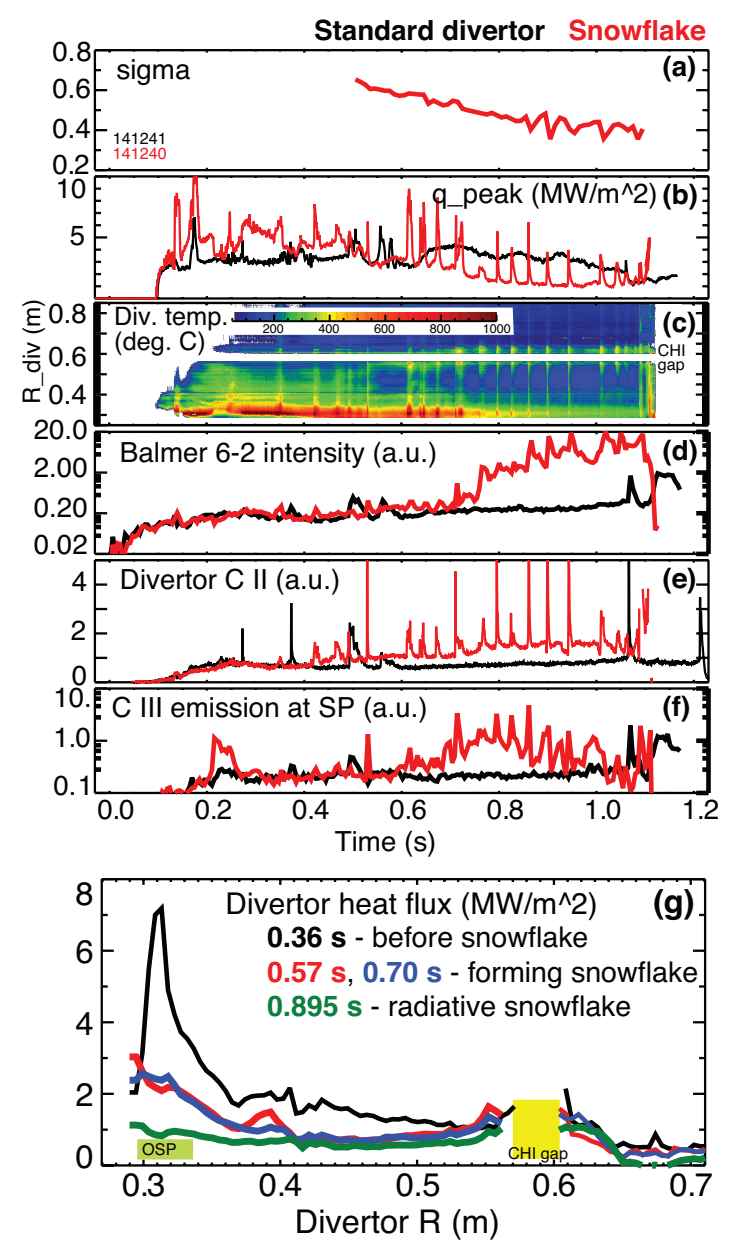

Figure 3: (a)-(f) - Divertor time traces of a standard divertor H-mode discharge (black lines) and SF-minus discharge (red lines) in NSTX: (a) Normalized null-point separation $\sigma$; (b) divertor peak heat flux from IR thermography; (c) divertor surface temperature from IR thermography in the SF-minus discharge; (d) Balmer $n=6-2$ line intensity in the strike point region; (e) Integrated divertor CII $\lambda 658.5 \mathrm{~nm}$ line brightness; (f) Divertor strike point C III $\lambda 407 \mathrm{~nm}$ brightness. (g) - Divertor heat flux profiles from IR thermography in the SF discharge (before, during, and after SF configuration formation). 


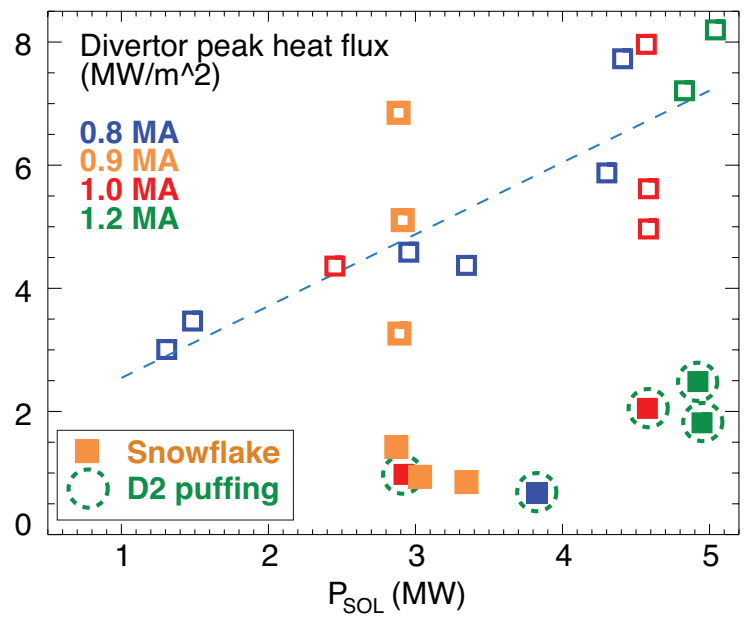

Figure 4: Divertor peak heat flux $q_{p e a k}$ in NSTX measured by IR thermography between ELMs in standard divertor discharges (open symbols) for a range of $I_{p}$ and $P_{S O L}$, and the reduced $q_{\text {peak }}$ in the radiative divertor discharges with $\mathrm{D}_{2}$ puffing (circled filled symbols) and the snowflake discharges without any $\mathrm{D}_{2}$ puffing (orange filled symbols). 


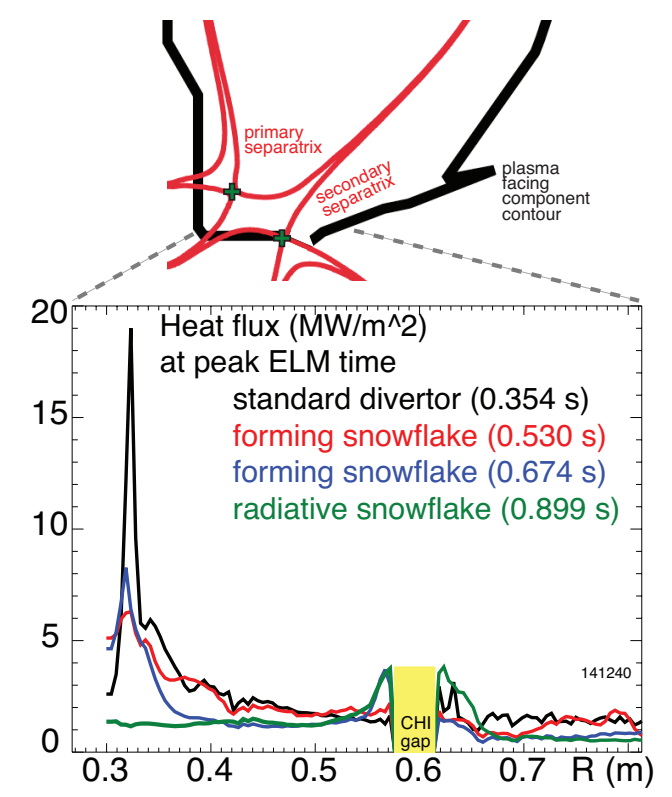

Figure 5: Divertor peak heat flux in NSTX measured by IR thermography at peak ELM times before and during the SF formation, as well as in the radiative SF phase. The upper inset shows two separatrix branches in the asymmetric SF-minus configuration. 